\title{
Descripción y análisis exploratorio de dos modelos de atención de los Trastornos de Espectro Autista en los niños y adolescentes en edad escolar del Área Mancha Centro.
}

Descriptive and exploratory analysis of two models of care for Autism Spectrum Disorder in children and adolescents in the Area "Mancha Centro".

\author{
Ana Maria Jiménez Pascual a , Cecilia Gracia Gallego o ${ }^{\text {, José María Tenías }}{ }^{\mathrm{c}}$, Pilar Fuertes Benei- \\ tez d, Carmen Abellán Maeso ${ }^{\mathrm{e}}$, Belén Cabezas Mateos ${ }^{\mathrm{f}}$, Jesús Ruiz Aranda ${ }^{\mathrm{g}}$.
}

\begin{abstract}
${ }^{a}$ Servicio de Psiquiatría y Salud Mental del Área Mancha Centro, ${ }^{b}$ Servicio de Psiquiatría y Salud Mental del Área Mancha Centro, ${ }^{c}$ Unidad de Apoyo a la Investigación del Hospital General Mancha Centro, Alcázar de San Juan, ${ }^{d}$ Unidad de Salud Mental Infanto Juvenil del Servicio de Psiquiatría y Salud Mental del Área Mancha Centro, ${ }^{e}$ Unidad de Salud Mental Infanto Juvenil del Servicio de Psiquiatría y Salud Mental del Área Mancha Centro, ${ }^{f}$ Unidad de Salud Mental Infanto Juvenil del Servicio de Psiquiatría y Salud Mental del Área Mancha Centro, ${ }^{8}$ Unidad de Salud Mental Infanto Juvenil del Servicio de Psiquiatría y Salud Mental del Área Mancha Centro.
\end{abstract}

Correspondencia: Ana María Jiménez.Pascual (salmendelin@sescam.jccm.es)

Recibido: 11/04/2011; aceptado con modificaciones: 27/06/2011

RESUMEN: Objetivo. Identificar y cuantificar si existen diferencias en el funcionamiento actual, características socio- familiares y datos del desarrollo entre los niños y adolescentes diagnosticados de TEA en edad escolar (de 3 a 16 años) del Área La Mancha Centro que asisten a centros de educación especial (EE) y los que asisten a hospital de día (HD).

Material y métodos. Estudio observacional trasversal, en el que se exploran las posibles diferencias entre los dos modelos de atención. Se seleccionaron los niños diagnosticados de TEA entre 3 y 16 años residentes en el Área Mancha Centro. Las pruebas realizadas valoraron el funcionamiento intelectual (Weschler, K-ABC de Kauffman), habilidades adaptativas (Vineland), habilidades lingüísticas (Peabody) y de las características del funcionamiento (C.A.R.S). La valoración de cada niño se realizó por un investigador independiente a los centros estudiados.

Resultados. Se reclutaron 40 casos, 31 varones y 9 mujeres (ratio 3,4:1) con una edad media de 10,9 años (rango de 3 a 16 años). 29 casos fueron atendidos exclusivamente en el HD y 11 en centros de EE. Los niños atendidos en el HD presentaron una mayor frecuencia de trastornos en el embarazo y parto y de antecedentes mórbidos personales y familiares. Los resultados de los test mostraron un mayor nivel en todas las áreas evaluadas en los niños atendidos en el $\mathrm{HD}$, siendo las diferencias estadísticamente significativas en el desarrollo lingüístico, en la conducta adap-
ABSTRACT: Objective. Identify and quantify possible differences in current functioning, social and family characteristics and data development among children and adolescents diagnosed with ASD school age (from 3 to 16 years) Area Centro La Mancha attending special schools (SS) and those attending hospital day (HD).

Material and methods. Observational study, which explores the differences between the two models of care. We selected children diagnosed with ASD between 3 and 16 years living in the area Mancha Centro. Tests assessed intellectual functioning (Weschler, ABC Kauffman), adaptive skills (Vineland), language skills (Peabody) and performance characteristics (CARS). The assessment of each child was conducted by an independent researcher.

Results. We recruited 40 patients, 31 males and 9 females (ratio $3,4: 1$ ) with a mean age of 10.9 years (range 3 to 16 years). 29 cases were treated exclusively in HD and 11 SS centers. Children attended in the HD had a higher frequency of disorders in pregnancy and childbirth and personal and family history morbid. The test results showed a higher level in all areas evaluated in children seen in HD, with statistically significant differences in language development, adaptative behavior and some areas of functioning measured by the CARS.

Conclusions. This preliminary study shows that 
tatvia y en algunas áreas de funcionamiento medidas por el CARS.

Conclusiones. Se muestra de forma preliminar que el modelo de atención recibido en el Hospital de Día se asocia a una tendencia a evolucionar de forma más favorable en los niños con TEA.

PALABRAS CLAVE: Autismo, hospital de día, tratamiento cognitivo-conductual, trastornos del espectro autista

\section{Introducción}

Dentro del término Trastornos Generalizados del Desarrollo (TGD) se incluye un grupo de trastornos con sintomatología de características autistas $(1,2)$.

En los últimos años se tiende a remplazar el término TGD por el de Trastornos del Espectro Autista (TEA), donde la palabra autismo suele emplearse como un término genérico para referirse a un concepto más amplio y representan un conjunto de cuadros caracterizados por la presencia de conductas repetitivas (estereotipias) y problemas en la comunicación e interacción social.

En la Clasificación Francesa de los Trastornos Mentales del Niño y el Adolescente (CFTMEA-R-2000) (3), aunque con denominaciones diferentes, las equivalencias en el diagnóstico son claras. Sí quisiéramos puntualizar que el diagnóstico de Disarmonía evolutiva (psicótica) y los Trastornos límites de Personalidad, pueden corresponder más claramente al concepto de TEA.

En la Clasificación Diagnóstica 0-3 R (4), se evita la utilización tanto del término Autismo como de psicosis en la categoría principal, hablando de Trastornos de la relación y la comunicación que incluye el Trastorno Multisistémico del Desarrollo (TMSD), que puede utilizarse exclusivamente en niños hasta los 3 años.

En la actualidad hay una diversificación de modelos para el tratamiento de los TEA. A pesar de ello, existe un posicionamiento político a favor de uno de los modelos. El grupo parlamentario catalán de CiU realizó una propuesta no de ley que insta al gobierno a "Proponer al Consejo Interterritorial del Sistema Nacional de Salud que encomiende un estudio para valorar la oportunidad de incluir, en la actualización de la cartera de servicios del Sistema Nacional de Salud que se opere, los tratamientos de terapia conductual destinados a las personas con trastornos del espectro autista, caso que su eficacia, efectividad y eficiencia estuvieran asegurados por la evidencia científica disponible". Esta propuesta, sin embargo ha sido rebatida por la mayor parte de asociaciones de profesionales de salud mental de niños y adolescentes, así como por asociaciones de familiares de personas con TEA.

Dentro de los diversos abordajes terapéuticos en nuestra área sanitaria hay claramente establecidas dos tendencias: a) Abordaje bajo vertiente pedagógica: 
ORIGINALES Y REVISIONES

Modelo cognitivo-conductual y b) Modelo psicodinámico y relacional: tratamiento en hospital de día.

"Desde el enfoque cognitivo, el autismo es considerado no una enfermedad mental, sino un trastorno del desarrollo que requiere una atención psicoeducativa en un ambiente estructurado. Las orientaciones centradas en la concepción neuropsicobiológica se sitúan en una causalidad lineal (unión directa entre causas y consecuencias) y su abordaje terapéutico se inscribe principalmente en las consecuencias patológicas que se deriven de las lesiones y déficit. El objetivo es paliar el déficit y aumentar las competencias socialmente utilizables. El abordaje educativo se plantea como única forma de intervención, ya que una educación adaptada puede disminuir los trastornos del comportamiento y desarrollar las capacidades útiles, a pesar de la persistencia del déficit fundamental. Se enfatiza el aprendizaje de habilidades comunicacionales, sociales, estimular la motivación y disminuir conductas desadaptadas. Se trabaja en conjunto con la escuela y los padres mediante abordajes estructurados, con método cognitivo conductual, preparando al niño y futuro adulto para integrarse en la comunidad". (5-8).

Dentro de este abordaje los niños y adolescentes pueden tener dos posibilidades de escolarización: en colegios de Educación Especial o bien en colegios ordinarios, con apoyos específicos.

Según el modelo psicodinámico y relacional, con tratamiento en hospital de día, se considera que los trastornos del espectro autista, por causas aun desconocidas (aunque posiblemente en la mayor parte de casos de origen multifactorial), son el efecto de un trastorno de la intersubjetividad primaria, siendo esta en el desarrollo normal esencial para la estructuración del psiquismo del niño (9). Se parte además de que las dificultades de integración de sensaciones, afectos y representaciones, provocan que persistan sensaciones no conectadas a procesos cognitivos y emocionales (no puede identificar, clasificar, nombrar, darles un sentido a sus sensaciones), pudiendo aparecer situaciones de mayor tensión ansiedades muy primitivas ligadas a sensaciones corporales (10), por lo que se evita la relación con el entorno intentando que este no cambie con actitudes de control.

Como señala Misés (11) el abordaje de estos niños "debe englobar una doble vertiente terapéutica y educativa que es preciso articular entre sí, en una aproximación multidimensional donde la preponderancia de dichas intervenciones variará según el caso y según las circunstancias”. Por ello también se utilizan técnicas congnitivas, pero siembre dentro de un marco relacional. En algunos casos, los hospitales de día parten de un trabajo fundamentalmente cognitivoconductual.

También dentro de este modelo existen dos posibilidades:

- Hospital de día a tiempo completo, donde los chicos durante gran parte del tratamiento no van a la escuela (12). 
- Hospital de Día a tiempo parcial o Unidad de Terapia Intensiva, donde los chicos combinan su estancia en el dispositivo clínico, acudiendo también a su colegio correspondiente donde a su vez reciben apoyo psicopedagógico (13).

En ambos casos, los niños menores de tres años suelen estar atendidos en Atención Temprana, con tratamientos fundamentalmente de estimulación del lenguaje y de psicomotricidad.

En el Área La Mancha Centro los dispositivos que existen, además de la Atención Temprana, son por una parte los colegios de educación especial y por otro el Hospital de Día a Tiempo parcial, de orientación psicodinámica y relacional. El que los niños acudan al colegio de educación especial o al hospital de día, en principio no depende de que tengan una mejor competencia lingüística, comportamental, y cognitiva.

Con este estudio, se pretende identificar y cuantificar si existen diferencias en la sintomatología actual, entre los niños y adolescentes en edad escolar (de 3 a 16 años) del Área La Mancha Centro con estos diagnósticos que asisten a centros de educación especial y los que asisten a hospital de día.

Así mismo se pretende describir e identificar si existen diferencias significativas entre las características socio-familiares de estos niños y adolescentes que acuden a estos dispositivos.

\section{Material y métodos}

Diseño. Se trata de un estudio observacional trasversal, eminentemente descriptivo, aunque también se exploran las posibles diferencias entre los dos modelos de atención.

\section{Sujetos de estudio}

Dentro del estudio, los criterios de inclusión han sido:

- Todos los sujetos desde los 3 años hasta los 16 años, edad escolar obligatoria, residentes en el área la Mancha Centro, con asistencia a colegios de educación especial, a escuelas ordinarias y/o al Hospital de Día de la Unidad de Salud Mental Infanto Juvenil (USMIJ) del área La Mancha Centro y con los siguientes diagnósticos de TEA (independiente de por quien fue diagnosticado):

* El Trastorno Autista (también el atípico), los Trastornos Generalizados del Desarrollo No Especificados, el Trastorno Desintegrativo de la Infancia, el Síndrome de Asperger y Otros Trastornos Generalizados del desarrollo, tal y como se definen en las clasificaciones europea y americana.

* Los Trastornos de la Relación y la Comunicación, denominados Tras- 
tornos Multisistémicos del Desarrollo (TMSD, Clasificación Diagnóstica: 0-3 R).

* Disarmonias evolutivas y Trastornos límites de la Personalidad (Clasificación francesa), equivalentes a Otros Trastornos de las Emociones y otros Trastornos del Desarrollo Psicológico.

Quedaron excluidos: el Síndrome de Rett, porque aunque es catalogado dentro de los TGD, es un trastorno con un componente genético y orgánico claro, con una trayectoria clínica muy diferente del resto de TEA y por tanto con abordajes terapéuticos diferentes. Por razones similares tampoco incluimos el "Trastorno hipercinético con retraso mental y movimientos estereotipados", perteneciente a la clasificación en la CIE10.

\section{Procedimiento}

Los datos sociofamiliares se recogieron de los datos de las historia clínicas (apartados A y B de la encuesta epidemiológica utilizada en la recogida de datos del hospital de día) de aquellos casos que han sido atendido en la USMIJ.

En los casos desconocidos para la USMIJ, se han utilizado los mismos apartados A y B de la encuesta epidemiológica, que han sido recogidos por personal de la USMIJ.

Una psicóloga independiente y no adscrita a ninguno de los dos modelos de atención evaludos, valoró todos los casos, sin conocer la procedencia de cada chico.

Las pruebas realizadas para evaluar las diferentes áreas han sido:

- Funcionamiento intelectual:

- Niños sin lenguaje. Inventario de Desarrollo Battelle/ Brunne- Lezine (14)

- Niños con lenguaje :

- Escalas Weschler $(15,16)$

- Batería K- ABC de Kauffman (17)

- Habilidades Adaptativas: Escala de Conducta Adaptativa de Vineland (18).

- Habilidades lingüísticas: Test Peabody (19)

- Evaluación de las características de funcionamiento: Escala de valoración del Autismo Infantil (CARS) (20)

\section{Grupos de análisis}

Se realizaron dos clasificaciones en relación al tipo de intervención/modelo terapéutico y educativo realizado en cada niño:

- Diferenciación entre el modelo de Hospital de día (HD) y el de Educación Especial (EE) puro (clasificación o modelo 1 de "educación especial pura") 
Se adscribió cada caso a uno de los dos grupos siguientes

- Hospital de día: si había sido atendido en este modelo o bien había permanecido en el mismo al menos 1 año (tiempo mínimo que se establece para poder tener eficacia terapéutica), independientemente de si había sido atendido o no en el modelo de educación especial.

- Educación especial puro. Solamente si el niño había sido atendido en este modelo de forma exclusiva o bien compartido con Hospital de día durante menos de un año.

- Diferenciación entre el modelo de Hospital de día y el de Educación Especial pura o mixta (clasificación o modelo 2 de "Hospital de día puro")

- Hospital de día puro: si había sido atendido exclusivamente en este modelo, fuera cual fuera el tiempo, sin educación especial.

- Educación especial pura/mixta. Si el niño había sido atendido en este modelo de forma exclusiva o bien compartido con Hospital de día.

Existe un tercer grupo de niños/as que están integrados en colegios ordinarios con apoyos, pero el $\mathrm{n}^{\circ}$ es tan escaso en nuestro área sanitaria que no ha sido posible realizar una comparación con los otros dos grupos.

\section{Estrategia de análisis}

Análisis descriptivo. Las variables cuantitativas se resumieron con medidas de tendencia central (media) y de dispersión (desviación estándar, DE) y las cualitativas con frecuencias absolutas y relativas expresadas como porcentajes.

Los contrastes entre los dos modelos de atención se realizaron con pruebas de Ji cuadrado o test exacto de Fisher (cualitativas) y con la prueba no paramétrica U de Mann-Whitney para las variables cuantitativas.

Se seleccionó como nivel de significación un riesgo alfa del 5\% $(\mathrm{p}<0,05)$

En el tratamiento estadístico de los datos se ha utilizado el programa SPSS para Windows (versión 18.0)

\section{Resultados}

Se seleccionaron 40 casos, 9 niñas $(22,5 \%)$ y 31 niños $(77,5 \%)$ con una edad media de 10,9 años (DE 3,7 años; rango de 3 a 16,8 años).

La atención de estos niños se realizó de forma mayoritaria en el Hospital de Día, con 35 casos $(87,5 \%)$ atendidos en esta área y 5 en educación especial según los criterios del modelo 1 . 
ORIGINALES Y REVISIONES

Los niños atendidos de forma exclusiva en el hospital de día fueron 29 $(72,5 \%)$ según la clasificación del modelo 2.

\section{Diagnósticos}

El diagnóstico predominante, $60 \%$, fue el de Otros Trastornos Generalizados del Desarrollo (F84.8 de la CIE 10) seguido con un 17\% de Otros Trastornos del Desarrollo Psicológico (F88). El 7,5\% corresponde a Otros Trastornos Generalizados del desarrollo no especificos (F84.9) y un 5\% tanto a Síndrome de Asperger (F84.5) como a Otros trastornos emocionales en la niñez. El 2'5\% son Trastorno Autista (F84.0) y otro 2,5\%, Otros trastornos Emocionales y del Comportamiento.

\section{Datos del desarrollo}

En las tabla 1 se aprecian los datos globales del embarazo y parto en ambos modelos. En el 57\% de casos ha habido alguna dificultad en el embarazo y/o el parto. Según la clasificación 1, todas las dificultades somáticas en el embarazo, así como el parto prolongado, cesáreas, parto prematuro y depresiones post parto, están en el grupo de HD.

Tabla 1

Problemas durante el embarazo y el parto en relación al modelo de atención (clasificación 1)

\begin{tabular}{|lc|ccc|ccc|}
\hline Variables & $\begin{array}{r}\text { Global } \\
(\mathbf{n}=\mathbf{4 0})\end{array}$ & $\begin{array}{l}\text { Hospital de Educación } \\
\text { Día (n=35) especial (n=5) }\end{array}$ & $\mathbf{p}$ & $\begin{array}{l}\text { Mospital de Educación } \\
\text { Día (n=29) especial (n=11) }\end{array}$ & p \\
\hline $\begin{array}{l}\text { Dificultades } \\
\text { en embarazo }\end{array}$ & $15(37,5 \%)$ & $14(40 \%)$ & $1(20 \%)$ & 0,63 & $13(44,8 \%)$ & $2(18,2 \%)$ & 0,16 \\
\hline $\begin{array}{l}\text { Dificultades } \\
\text { en el parto }\end{array}$ & $14(35 \%)$ & $13(37,1 \%)$ & $1(20 \%)$ & 0,64 & $11(37,9 \%)$ & $3(27,3 \%)$ & 0,72 \\
\hline $\begin{array}{l}\text { Alguna dificultad } \\
\text { en el embarazo y/o parto }\end{array}$ & $23(57,5 \%)$ & $21(60 \%)$ & $2(40 \%)$ & 0,63 & $18(62,1 \%)$ & $5(45,5 \%)$ & 0,48 \\
\hline
\end{tabular}

En la tabla 2 observamos los datos relativos al desarrollo del niño: El 40\% de los casos ha presentado algún antecedente mórbido personal (hospitalización perinatal, Intervención quirúrgica, hospitalización no quirúrgica, accidentes, crisis convulsivas, frecuencia alta enfermedades).

El 45\% de los niños han tenido lactancia materna. El 65\% caminó antes de los 18 meses, perteneciendo la mayoría según la clasificación 1, al grupo de HD. Con respecto al control de esfínteres, solo el 27,5\% lo ha adquirido antes de los 5 años.

El 27,5\% de los niños ha tenido trastornos del sueño durante el primer año de vida. 
Tabla 2

Datos del desarrollo

\begin{tabular}{|c|c|c|c|c|c|c|c|}
\hline Variables & $\begin{array}{l}\text { Global } \\
(n=40)\end{array}$ & $\begin{array}{l}\text { Hospital de } \\
\text { Día }(n=35)\end{array}$ & $\begin{array}{l}\text { Modelo 1 } \\
\text { Educación } \\
\text { especial }(n=5)\end{array}$ & $\mathbf{p}$ & $\begin{array}{l}\text { Hospital de } \\
\text { Día }(n=29)\end{array}$ & $\begin{array}{c}\text { Modelo 2 } \\
\text { Educación } \\
\text { especial (n=11) }\end{array}$ & $\mathbf{p}$ \\
\hline $\begin{array}{l}\text { Algún antecedente mórbido } \\
\text { familiar }(A M P)\end{array}$ & $16(40 \%)$ & $14(40 \%)$ & $2(40 \%)$ & 1 & $12(41,4 \%)$ & $4(36,4 \%)$ & 0,78 \\
\hline Lactancia natural & $18(45 \%)$ & $17(48,6 \%)$ & $1(20 \%)$ & 0,36 & $14(48,3 \%)$ & $4(36,4 \%)$ & 0,72 \\
\hline $\begin{array}{l}\text { Caminó antes de los } \\
18 \text { meses }\end{array}$ & $26(65 \%)$ & $24(68,6 \%)$ & $2(40 \%)$ & 0,32 & $21(72,4 \%)$ & $5(45,5 \%)$ & 0,15 \\
\hline $\begin{array}{l}\text { Control esfinteres } \\
\text { antes } 5 \text { años }\end{array}$ & $11(27,5 \%)$ & $9(25,7 \%)$ & $2(40 \%)$ & 0,60 & $7(24,1 \%)$ & $4(36,4 \%)$ & 0,46 \\
\hline $\begin{array}{l}\text { Trastornos del sueño en el } \\
\text { primer año de vida }\end{array}$ & $11(27,5 \%)$ & $10(28,6 \%)$ & $1(20 \%)$ & 1 & $8(27,6 \%)$ & $3(27,3 \%)$ & 1 \\
\hline Nacimiento de un hermano & $8(20 \%)$ & $6(17,1 \%)$ & $2(40 \%)$ & 0,26 & $5(17,2 \%)$ & $3(27,3 \%)$ & 0,66 \\
\hline Otros cambios familiares & $5(12,5 \%)$ & $5(14,3 \%)$ & - & 1 & $5(17,2 \%)$ & - & 0,30 \\
\hline Abortos previos & $7(17,5 \%)$ & $7(20 \%)$ & - & 0,57 & $7(24,1 \%)$ & - & 0,16 \\
\hline Enfermedad crónica padres & $8(20 \%)$ & $8(22,9 \%)$ & - & 0,56 & $8(27,6 \%)$ & - & 0,08 \\
\hline Enfermedad orgánica & $5(12,5 \%)$ & $5(14,3 \%)$ & - & 1 & $3(10,3 \%)$ & $2(18,2 \%)$ & 0,60 \\
\hline
\end{tabular}

En relación a cambios familiares y episodios intercurrentes en la vida del niño, en la clasificación 1, solo existen Otros cambios familiares (14,3\%) en el HD. Así mismo solo aparecen en este grupo de HD, abortos previos (20\%), enfermedades crónicas de los padres $(22,9 \%)$, enfermedad psíquica de hermanos $(2,9 \%)$ y el $14,3 \%$ han padecido enfermedad orgánica.

El 89,2\% han adquirido el lenguaje; en la clasificación 1, en el grupo de HD lo han adquirido el 90,9\% y en el grupo de EE, el $75 \%$.

\section{Situación sociofamiliar}

La instrucción de los padres fue elemental en el 15\% de los casos (tanto para el padre como para la madre) y más de la mitad de los casos tenían una educación de grado medio (bachiller, formación profesional). Solamente el 7\% habían cursado estudios superiores. El nivel de instrucción no fue diferente según los dos modelos de clasificación analizados.

En 7 niños $(17,5 \%)$ se constató un antecedente paterno o materno de alteración psicopatológica. Según la clasificación 1, todos estos casos fueron atendidos en el Hospital de Día (Tabla 3).

La mayoría de los procesos psicopatológicos eran cuadros de ansiedad y/o depresión tanto en el padre como en la madre. 
Tabla 3

Antecedentes familiares

\begin{tabular}{|c|c|c|c|c|c|c|c|}
\hline Variables & $\begin{array}{l}\text { Global } \\
(n=40)\end{array}$ & \multicolumn{2}{|c|}{\begin{tabular}{|l} 
Modelo 1 \\
Hospital de Educación \\
Día $(n=35)$ especial $(n=5)$ \\
\end{tabular}} & $\mathbf{p}$ & \multicolumn{2}{|c|}{\begin{tabular}{|c} 
Modelo 2 \\
Hospital de Educación \\
Día $(n=29)$ especial $(n=11)$
\end{tabular}} & \multirow[t]{2}{*}{$\mathbf{p}$} \\
\hline $\begin{array}{l}\text { Niños con padre } y / o \\
\text { madre con trastorno } \\
\text { psicopatológico }\end{array}$ & $7(17,5 \%)$ & $7(20 \%)$ & 0 & 0,56 & $6(20,7 \%)$ & $1(9,1 \%)$ & \\
\hline $\begin{array}{l}\text { Niños con madre con } \\
\text { trastorno psicopatológico }\end{array}$ & $5(12,5 \%)$ & $5(24,3 \%)$ & 0 & 1 & $5(17,2 \%)$ & 0 & 0,30 \\
\hline $\begin{array}{l}\text { Niños con padre con } \\
\text { trastorno psicopatológico }\end{array}$ & $2(5 \%)$ & $2(5,7 \%)$ & 0 & 1 & $1(3,4 \%)$ & $1(9,1 \%)$ & 0,48 \\
\hline
\end{tabular}

\section{Evaluación del funcionamiento}

En este apartado, el número de la muestra es menor, al no haber aun terminado de valorar a todos los chicos. Varía así mismo el número de casos en las diferentes áreas exploradas, por el mismo motivo.

\section{Funcionamiento intelectual}

En este apartado no se pueden establecer comparaciones entre ambos modelos, ya que tan solo en un caso de EE (clasificación 2), se ha podido realizar la prueba; en los demás, bien por falta de lenguaje, bien por falta de comprensión de lo que se les demandaba, ha sido imposible realizarla, ni siquiera el Brunne Lezine, ni el Battelle, que aun sin lenguaje, si tiene que haber una cierta actitud de colaboración, con un mínimo de comprensión.

El $\mathrm{n}^{\circ}$ de la muestra es de 13 casos, 12 el HD y 1 de EE. La media del CI en el grupo del HD ha sido de 78,6 $\pm 15,3$, con un rango de 53 a 110, lo que indica que la medía de chicos evaluados hasta ahora, están dentro de un CI normal- bajo

\section{Habilidades adaptativas}

En la Escala de conducta adaptativa de Vineland, en cada área explorada existen tres niveles de funcionamiento: bajo (puntuación de 20 a 69), moderado (puntuación de 70 a 80) y adecuado (puntuación de 80 a 100).

En ambas clasificaciones (tabla 4) los casos de EE están en funcionamiento de nivel bajo. Los casos de HD, alrededor de un $50 \%$ tienen un funcionamiento moderado en casi todas las áreas.

\section{Habilidades lingüísticas}

En este apartado la muestra ha sido de 26 casos (tabla 5). En la clasificación 1, ninguno de los tres casos de EE tenía lenguaje, por lo que la puntuación fue 0 , siendo imposible por ese motivo hallar la edad equivalente. 
Tabla 4

Interpretación cualitativa en los dominios de la escala de conducta adaptativa de Vineland en relación al modelo de atención

\begin{tabular}{|c|c|c|c|c|c|c|c|}
\hline Variables & $\begin{array}{l}\text { Global } \\
(n=40)\end{array}$ & $\begin{array}{c}\text { Hospital de } \\
\text { Día }(n=19)\end{array}$ & $\begin{array}{c}\text { Modelo 1 } \\
\text { Educación } \\
\text { especial }(n=3)\end{array}$ & $\mathbf{p}$ & $\begin{array}{l}\text { Hospital de } \\
\text { Día }(n=16)\end{array}$ & $\begin{array}{c}\text { Modelo 2 } \\
\text { Educación } \\
\text { especial }(n=6)\end{array}$ & $\mathbf{p}$ \\
\hline Comunicación & & & & 0,18 & & & 0,02 \\
\hline Bajo & $11(50 \%)$ & $8(42,1 \%)$ & $3(100 \%)$ & & $5(31,3 \%)$ & $6(100 \%)$ & \\
\hline Moderado & $8(36,4 \%)$ & $8(42,1 \%)$ & - & & $8(50,0 \%)$ & - & \\
\hline Adecuado & $3(13,6 \%)$ & $3(15,8 \%)$ & - & & $3(18,8 \%)$ & - & \\
\hline Habilidades de la vida diaria & & & & 0,18 & & & 0,04 \\
\hline Bajo & $11(50 \%)$ & $8(42,1 \%)$ & $3(100 \%)$ & & $5(31,3 \%)$ & $6(100 \%)$ & \\
\hline Moderado & $6(27,3 \%)$ & $6(31,6 \%)$ & - & & $6(37,5 \%)$ & - & \\
\hline Adecuado & $5(22,7 \%)$ & $5(26,3 \%)$ & - & & $5(31,3 \%)$ & - & \\
\hline Socialización & & & & 0,08 & & & 0,04 \\
\hline Bajo & $9(40,9 \%)$ & $6(31,6 \%)$ & $3(100 \%)$ & & $4(25 \%)$ & $5(83,3 \%)$ & \\
\hline Moderado & $10(45,5 \%)$ & $10(52,6 \%)$ & - & & $9(56,3 \%)$ & $1(16,7 \%)$ & \\
\hline Adecuado & $3(13,6 \%)$ & $3(15,8 \%)$ & - & & $3(18,8 \%)$ & - & \\
\hline $\begin{array}{l}\text { Comportamiento adaptativo } \\
\text { compuesto }\end{array}$ & & & & 0,12 & & & 0,007 \\
\hline Bajo & $10(45,5 \%)$ & $7(36,8 \%)$ & $3(100 \%)$ & & $4(25 \%)$ & $6(100 \%)$ & \\
\hline Moderado & $10(45,5 \%)$ & $10(52,6 \%)$ & - & & $10(62,5 \%)$ & - & \\
\hline Adecuado & $2(9,1 \%)$ & $2(10,5 \%)$ & - & & $2(12,5 \%)$ & - & \\
\hline
\end{tabular}

Tabla 5

Resultados de la escala Peabody en relación al modelo de atención (clasificación 1)

\begin{tabular}{|c|c|c|c|c|c|c|c|}
\hline \multirow{2}{*}{$\begin{array}{l}\text { Variables } \\
\text { Dominio }\end{array}$} & \multirow{2}{*}{$\begin{array}{c}\begin{array}{c}\text { Global } \\
(\mathbf{n}=\mathbf{2 6})\end{array} \\
64,1 \pm 52,3\end{array}$} & \multicolumn{2}{|c|}{\begin{tabular}{|l} 
Modelo 1 \\
Hospital de Educación \\
Día $(n=23)$ especial $(n=3)$
\end{tabular}} & \multirow{2}{*}{$\frac{\mathbf{p}^{*}}{0,008}$} & \multirow{2}{*}{\multicolumn{2}{|c|}{ 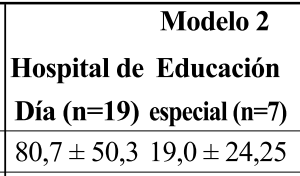 }} & \multirow{2}{*}{$\frac{\mathbf{p}^{*}}{0,007}$} \\
\hline & & $72,4 \pm 49,8$ & 0 & & & & \\
\hline Edad equivalente, $n=20$ & $7,8 \pm 3,7$ & $7,8 \pm 3,7$ & - & & $8,3 \pm 3,7$ & $4,6 \pm 0,6$ & 0,15 \\
\hline $\begin{array}{l}\text { Ratio edad equivalentel } \\
\text { edad real, } n=20\end{array}$ & $0,80 \pm 0,35$ & $0,80 \pm 0,35$ & - & & $0,87 \pm 0,32$ & $0,36 \pm 0,08$ & 0,007 \\
\hline
\end{tabular}

* Ude Mann-Whitney

Evaluación de las características de funcionamiento

En esta prueba, el CARS, las diferentes áreas exploradas tienen 4 puntuaciones de evaluación y sus intermedios, si el niño está entre dos niveles. La puntuación 1 indica la mayor normalidad, mientras que la puntuación 4 es la de mayor gravedad.

En la clasificación 1, al solo haber un solo caso de EE, no tomaremos en cuenta los resultados. 
En la clasificación 2 (tabla 6), en la "impresión general" se obtienen puntuaciones de 1,63 para el grupo de HD y 2,83 para el de EE.

Correlación del tiempo de atención en el Hospital de día y las escalas de Peabody y CARS

Mediante un análisis de correlación observamos que a mayor tiempo del niño en el HD se tiende a obtener mayores puntuaciones en la escala de Peabody $(r=0,32 ; p=0,14)$ y menores en el CARS $(r=-0,17 ; p=0,49)$.

Tabla 6

Valores de la escala C.A.R.S. (clasificación 2)

\begin{tabular}{|l|cccc|}
\hline Variables & $\begin{array}{c}\text { Global } \\
(\mathbf{n}=\mathbf{2 0})\end{array}$ & $\begin{array}{c}\text { Hospital de Día } \\
(\mathbf{n}=\mathbf{1 7})\end{array}$ & $\begin{array}{c}\text { Educación } \\
\text { especial } \mathbf{( n = 3 )}\end{array}$ & $\mathbf{p}^{*}$ \\
\hline Relación con la gente & $2,03 \pm 0,66$ & $2,06 \pm 0,67$ & $1,83 \pm 0,76$ & 0,82 \\
Imitación & $1,25 \pm 0,73$ & $1,18 \pm 0,73$ & $1,67 \pm 0,76$ & 0,15 \\
Respuesta emocional & $1,50 \pm 0,43$ & $1,50 \pm 0,43$ & $1,50 \pm 0,50$ & 0,64 \\
Uso del cuerpo & $1,68 \pm 0,77$ & $1,47 \pm 0,62$ & $2,38 \pm 0,95$ & 0,099 \\
\hline Uso del objeto & $1,75 \pm 0,87$ & $1,76 \pm 0,85$ & $1,67 \pm 1,15$ & 0,86 \\
Adaptación al cambio & $1,08 \pm 0,18$ & $1,06 \pm 0,17$ & $1,16 \pm 0,29$ & 0,62 \\
Respuesta visual & $1,53 \pm 0,66$ & $1,47 \pm 0,60$ & $2,13 \pm 1,03$ & 0,20 \\
Respuesta auditiva & $1,25 \pm 0,55$ & $1,21 \pm 0,50$ & $1,63 \pm 0,75$ & 0,36 \\
\hline Uso y respuesta gusto, olfato, tacto & $1,28 \pm 0,60$ & $1,24 \pm 0,56$ & $1,50 \pm 0,87$ & 0,69 \\
Miedo o nerviosismo & $1,30 \pm 0,52$ & $1,26 \pm 0,53$ & $1,50 \pm 0,50$ & 0,36 \\
Comunicación verbal & $2,23 \pm 0,94$ & $2,12 \pm 0,91$ & $2,83 \pm 1,04$ & 0,22 \\
Comunicación no verbal & $1,35 \pm 0,67$ & $1,29 \pm 0,66$ & $1,67 \pm 0,76$ & 0,31 \\
\hline Nivel de actividad & $1,53 \pm 0,68$ & $1,41 \pm 0,62$ & $2,17 \pm 0,76$ & 0,09 \\
Nively consistencia respuesta intelectual & $1,84 \pm 0,67$ & $1,81 \pm 0,72$ & $2,00 \pm 0$ & 0,42 \\
Impresión general & $1,68 \pm 0,77$ & $1,63 \pm 0,74$ & $2,83 \pm 1,17$ & 0,03 \\
\hline
\end{tabular}

\section{Discusión}

Con este estudio observacional y exploratorio hemos comprobado como, en general, en los niños con Trastorno del Espectro Autista, se aprecian diferencias entre aquellos casos tratados en el Hospital de Día y los que acuden a los colegios de Educación Especial. Aunque en la mayoría de los casos estas diferencias no son estadísticamente significativas, si pueden ser relevantes a nivel clínico, observándose unos mejores resultados en todas las áreas en el grupo del HD.

El número de casos detectados hasta la actualidad es de 40, lo que supone una menor prevalencia de la esperada. Si aplicamos la prevalencia estimada en otros estudios americanos, europeos y españoles (21-23) se deberían haber detectado un 
total de 220 casos de TEA en nuestro área de atención. Este punto sería importante constatarlo, pues si bien con este estudio no podemos aún estimar la verdadera prevalencia en el área, son pocos los centros que quedan por analizar por lo que prevemos que se añadirán relativamente pocos casos a los ya detectados, indicando una baja prevalencia en esta zona.

La relación varón/ mujer es de 3,4:1, la cual es similar a la estimada en los estudios europeos (24).

El mayor porcentaje de casos atendidos en HD podemos interpretarlo como un indicador de la buena implantación de este dispositivo en la zona.

En relación a los datos del desarrollo, los niños atendidos en el HD presentaron una mayor frecuencia de trastornos en el embarazo y parto y de antecedentes mórbidos personales. También nos llama la atención que solo aparecen en el grupo de HD, abortos previos, enfermedades crónicas de los padres, enfermedad psíquica de hermanos y otros cambios familiares. Ambos grupos de atención no fueron diferentes en la mayor parte de los datos sociofamiliares recogidos aunque observamos que prácticamente todos los casos con antecedente paternos de psicopatología pertenecen al HD.

Estos datos pueden ser clínicamente importantes y aunque hay que confirmar a nivel epidemiológico su posible papel causal en futuros estudios comparándolos con un grupo de controles sanos, si que podemos al menos apuntar una mayor frecuencia de factores mórbidos en los niños atendidos en el HD.

La mayoría de los que han tenido lactancia materna corresponden al grupo de HD. Aunque la diferencia no es estadísticamente significativa, clínicamente si puede ser relevante y sería interesante seguir constatando este dato en otras muestras, ya que podría ser una de las áreas que nos permita estudiar el papel de las interacciones tempranas en el desarrollo de este tipo de trastornos, aunque nunca como un dato aislado. Lo mismo ocurre con los problemas de alimentación, donde todos los trastornos encontrados están en el grupo de HD.

Alrededor del $70 \%$ en ambos grupos, no alcanzaron el control de esfínteres antes de los 5 años; en ambas clasificaciones hay un mayor porcentaje de casos de EE que lo han adquirido antes de esa edad y aunque estas diferencias no fueron estadísticamente significativas, de confirmarse, podrían indicarnos una mayor eficacia en los métodos utilizados en EE.

En el momento de la realización de este estudio, la mayoría de niños habían adquirido el lenguaje, siendo el porcentaje algo mayor en el grupo de HD.

El 27,5\% de trastornos del sueño en el primer año de vida nos parece un dato significativo que habría que constatar con estudios comparativos con grupos de la población general, ya que podría ser un signo de alarma a tener en cuenta.

No hemos encontrado diferencias en relación al nivel de instrucción de los padres (utilizada como una variable aproximativa a la clase social) en ambos gru- 
ORIGINALES Y REVISIONES

pos de atención. En este sentido, Wing (1993) (24) en una cuidadosa revisión de la prevalencia del autismo en relación a la clase social y al sexo, encuentra que de 16 estudios sólo 9 registraron la clase social, y la mayoría de ellos no encontraron diferencias respecto a esta variable con la población general.

Después de una búsqueda exhaustiva no hemos encontrado estudios que contraten ambos modelos de atención en la literatura.

En la evaluación del funcionamiento actual de los casos podemos destacar:

- La media del CI en el grupo del HD indica que están dentro de un CI normal- bajo. Teniendo en cuenta que el autismo está asociado con retraso mental $(\mathrm{CI}<70)$ en el 66-88\% de los casos en los estudios de población, los resultados del HD si se confirman al final del estudio, puede ser un importante indicador de los beneficios de este tipo de dispositivo terapéutico.

- En las habilidades lingüísticas las diferencias son significativas en ambas clasificaciones, tanto en las puntuaciones como en la ratio de edad equivalente/ edad real, siendo más favorable en los chicos del HD. Estos datos son importantes, pues si bien la mayoría de niños de ambos grupos habían adquirido el lenguaje, como se ha visto anteriormente, interesa además la capacidad de comunicación y de expresión amplia, lo que podría estar mejor reflejado en este apartado.

Se ha utilizado el Peabody en niños con autismo en otros estudios (25-26). En ambos trabajos, los niveles del test fueron similares a los de los niños de nuestro estudio en el HD, incluso algo mayores. Esto se debe a que el criterio de selección en ambos estudios requería de partida de una mínima capacidad lingüística por parte del niño para completar todos las pruebas a las que fueron sometidos. En nuestro caso, entre los niños atendidos en EE ninguno pudo completar el test de Peabody.

- En la evaluación de la conducta adaptativa, las diferencias son significativas. Independientemente del tipo de clasificación analizado, los casos de EE están en funcionamiento de nivel bajo. Para los niños del HD, en todas las áreas más de la mitad se sitúan en un nivel de funcionamiento moderado o adecuado, siendo máximo en las áreas de socialización y comportamiento adaptativo compuesto. En un estudio realizado en Yale y Michigan (27) los autores utilizan la escala de Vineland en niños autistas definidos de alto funcionamiento encontrando valores similares a los nuestros (27). En otro estudio canadiense (28), en el que comparan dos grupos de niños (con autismo y con retraso mental) los afectados de TEA mostraron unos resultados en el test de Vineland por debajo de los 70 puntos (correspondiente a un nivel funcional moderado-bajo).

- En las características de funcionamiento según la escala C.A.R.S., en la "impresión general" observamos una diferencia estadísticamente significativa entre ambos grupos, estando el grupo de HD próximo a la normalidad. Estos niveles son más favorables que los encontrados en otros estudios que utilizan la misma escala en niños con TEA con diferentes niveles cognitivos (28). 
Como se comentó en la introducción, el que los niños acudan al colegio de educación especial o al hospital de día no depende de una mejor competencia lingüística, comportamental, y/o cognitiva. Por ahora no hemos podido consensuar un protocolo que permita la indicación más idónea, ya que ambos modelos insisten en la importancia de su intervención desde el momento que es detectado el problema. Durante el tratamiento en HD, en algunos niños cuya evolución es más tórpida, si hay un cambio de escolaridad, dejan de acudir a este dispositivo y se escolarizan en el CE correspondiente, aunque siguen en tratamiento en la USMIJ.

Nuestro estudio presenta algunas limitaciones que merecen un comentario. Por un lado, se trata de un estudio observacional y por tanto no podemos descartas la presencia de sesgos tanto en la selección como en la recogida de la información en ambos grupos. Sin embargo hemos seguido de forma estricta un protocolo de selección y recogida de datos y el principal observador en la valoración de los pacientes estuvo enmascarado en cuanto a la procedencia, en relación al modelo de atención, de los niños. También el bajo número de casos analizados hace que el estudio tenga una baja sensibilidad (potencia estadística) para detectar diferencias que pueden llegar a ser clínicamente relevantes. En un futuro cercano podremos llegar a reclutar al menos 20 casos más por lo que algunos contrastes podrán llegar a ser además de clínicamente relevantes, también estadísticamente significativos.

Como propuestas de investigación creemos que sería interesante contrastar algunas de las características de estos niños (sobre todo los de desarrollo y sociofamiliares) con los de un grupo comparable de la población general para poder proponer posible hipótesis etiológicas en el desarrollo de este tipo de patología. Así mismo, sería relevante poder comparar algunos de los resultados desde el inicio de la atención en estos dispositivos, con un diseño longitudinal en panel que nos permita dilucidar las mejoras individuales a lo largo del tiempo.

En resumen, en este estudio mostramos de forma preliminar las características más relevantes de un grupo de niños con TEA que comparamos en relación con el modelo de atención recibido con una tendencia a evolucionar de forma más favorable en el caso de haber sido atendido de forma exclusiva o parcialmente en una unidad de Hospital de Día. 


\section{ORIGINALES Y REVISIONES}

\section{BIBLIOGRAFÍA:}

(1) Clasificación estadística internacional de enfermedades y problemas relacionados con la salud. Organización Mundial de la Salud. Trastornos mentales y del comportamiento. 10 ${ }^{\mathrm{a}}$ Ed (CIE-10). Ginebra: Meditor; 1993.

(2) Manual diagnóstico y estadístico de los trastornos mentales. Texto revisado (DSM-IV-TR). Barcelona, Editorial Masson, S.A; 2002.

(3) Classification Française des Troubles Mentaux de l'Enfant et de l'Adolescent (CFTMEA-R2000). Versión castellana de la AFAPSAM. Buenos Aires: Editorial Polemos; 2004.

(4) National Center for Clinical Infant Programs. Cero a tres. Clasificación Diagnóstica de la salud mental y los desórdenes en el desarrollo de la infancia y la niñez temprana. CD: 0-3. Buenos Aires: Paidós; 1997.

(5) Guía De Diagnostico Y Tratamiento De Los Trastornos Del Espectro Autista

(6) Gillberg, C. Prevalencia del autismo y de las alteraciones del espectro autista. Publicación en Internet en Center Londres 94.

(7) Diez-Cuervo A, Muñoz-Yunta JA, Fuente-Biggi J, Canal-Bedia R, Idiazábal- Aletxa MA, Ferrari-Arroyo MJ, et al. Guía de buena práctica para el diagnóstico de los trastornos del espectro autista. Rev Neurol 2005; 41 (5): 299-310.

(8) Fuentes-Biggi J, Ferrari-Arroyo MJ, Boada-Muñoz L. Touriño-Aguilera E, Artigas-Pallarés J, Belinchón-Carmona M, et al. Guía de buena práctica para el tratamiento de los trastornos del espectro autista. Rev Neurol 2006; 43: 425-38.

(9) Filippo Muratori. El autismo como efecto de un trastorno de la intersubjetividad primaria. Revista de Psicopatología y Salud Mental del niño y adolescente. No 12 . Noviembre 2008.

(10) Winnicott D.W. - Desarrollo emocional primitivo. En: Escritos de Pediatría y Psicoanálisis. Ed. Laia. 1979.

(11) Misés R. Pautas orientadoras en el tratamiento de la psicosis autísticas. En Parquet PhJ, Bursztejn C, Golse B, Editores. Autismo: Cuidados, educación y tratamiento. Barcelona: Masson S.A; 1992 .p. 17-31.

(12) Alcamí M. El tratamiento de la psicosis en hospitales de día. Cuadernos de psiquiatría y psicoterapia del niño y adolescente 2005; 39/40: 5-30.19-

(13) Jiménez A. Estructuras clínicas intermedias en la psicosis infantil. Rev Asoc Esp Neuropsiq 1999; 69: 151-59.

(14) Josse D. Escala de desarrollo psicomotor de la primera infancia Brunet- Lezine revisión 1997.

(15) Wechsler D. WPPSI: Escala de Inteligencia de Wechsler para preescolar y primaria. 7 ed. Madrid: TEA Ediciones; 2006.

(16) Wechsler D. Escala de Inteligencia de Wechsler para niños (WISC-IV). Madrid: TEA Ediciones; 2005.

(17) Kaufman AS, Kaufman NL. K-ABC: Batería de evaluación de Kaufman para niños. 2 ed. Madrid: TEA Ediciones; 2005.

(18) Sparrow SS, Cichetti D, Balla CA. Vineland Adaptive behaviour scales-2nd edition manual. Minneapolis MN,NCS Pearosn Inc.2005

(19) Dunn LIM, Dunn LM, Arribas D. PPVT-III: Peabody: Test de vocabulario en imágenes. Madrid: TEA Ediciones; 2006. 
(20) Escala de valoración del Autismo Infantil (C.A.R.S) 2002.

(21) Chakrabarti S. and Fombonne E. Pervasive Developmental Disorders in Preschool Children: Confirmation of High Prevalence. Am J Psychiatry 2005; 162: 1133-41.

(22) Baron-Cohen S, Scott FJ, Allison C, Williams J, Bolton P, Matthews FE, et al. Prevalence of autism-spectrum conditions: UK school-based population study. Br J Psychiatry. 2009;194(6):500-9.

(23) Sánchez-Valle E, Posada M, Villaverde-Hueso A, Touriño E, Ferrari-Arroyo MJ, Boada L, et al. Estimating the burden of disease for autism spectrum disorders in Spain in 2003. J Autism Dev Disord. 2008;38(2):288-96.

(24) Wing L. The continuum of autistic characteristics. En: Schopler E, Mesibov G, Ed. Diagnosis and Assessment in Autism. New York, NY: Plenum Press; 1988 .p. 1-10.

(25) McGrew S, Malow BA, Henderson L, Wang L, Song Y, Stone WL. Developmental and behavioral questionnaire for autism spectrum disorders. Pediatr Neurol. 2007; 37(2):108-16.

(26) Condouris K, Meyer E, Tager-Flusberg H. The relationship between standardized measures of language and measures of spontaneous speech in children with autism. Am J Speech Lang Pathol. 2003; 12(3):349-58.

(27) Klin A, Saulnier CA, Sparrow SS, Cicchetti DV, Volkmar FR, Lord C. Social and communication abilities and disabilities in higher functioning individuals with autism spectrum disorders: the Vineland and the ADOS. J Autism Dev Disord. 2007; 37(4): 748-59.

(28) Perry A, Flanagan HE, Dunn Geier J, Freeman NL. Brief report: the Vineland Adaptive Behavior Scales in young children with autism spectrum disorders at different cognitive levels. J Autism Dev Disord. 2009; 39(7):1066-78. 